\title{
PTPRQ as a potential biomarker for idiopathic normal pressure hydrocephalus
}

\author{
YUKI NAGATA $^{1}$, MASAHIKO BUNDO ${ }^{2}$, SAIKO SUGIURA $^{3}$, MASAHIRO KAMITA $^{4}$, MASAYA ONO $^{4}$, \\ KOTARO HATTORI $^{5}$, SUMIKO YOSHIDA ${ }^{5}$, YU-ICHI GOTO ${ }^{5}$, KATSUYA URAKAMI $^{6}$ and SHUMPEI NIIDA $^{1}$ \\ ${ }^{1}$ Medical Genome Center, National Center for Geriatrics and Gerontology; Departments of ${ }^{2}$ Experimental Neuroimaging and \\ ${ }^{3}$ Otolaryngology, National Center for Geriatrics and Gerontology, Obu, Aichi 474-8511; ${ }^{4}$ Division of Chemotherapy and \\ Clinical Research, National Cancer Center Research Institute, Chuo-ku, Tokyo 104-0045; ${ }^{5}$ Medical Genome Center, \\ National Center of Neurology and Psychiatry, Kodaira, Tokyo 187-8551; ${ }^{6}$ Department of Biological Regulation, School of \\ Health Science, Faculty of Medicine, Tottori University, Yonago, Tottori 683-8503, Japan
}

Received November 7, 2016; Accepted July 7, 2017

DOI: $10.3892 / \mathrm{mmr} .2017 .7015$

\begin{abstract}
Idiopathic normal pressure hydrocephalus (iNPH) is caused by the accumulation of cerebrospinal fluid (CSF) and is characterized by gait disturbance, urinary incontinence, and dementia. iNPH dementia is treatable by shunt operation; however, since the cognitive symptoms of iNPH are often similar to those of other dementias, including Alzheimer's disease (AD), accurate diagnosis of iNPH is difficult. To overcome this problem, the identification of novel diagnostic markers to distinguish iNPH and AD is warranted. Using comparative proteomic analysis of CSF from patients with iNPH and AD, protein tyrosine phosphatase receptor type Q (PTPRQ) was identified as a candidate biomarker protein for discriminating iNPH from AD. ELISA analysis indicated that the PTPRQ concentration in the CSF was significantly higher in patients with iNPH compared with those with AD. In addition, the PTPRQ concentration in the CSF of non-responders to shunt operation (SNRs) tended to be relatively lower compared with that in the responders. PTPRQ may be a useful biomarker for discriminating between patients with iNPH and AD, and may be a potential companion biomarker to identify SNRs among patients with iNPH. Additional large-scale analysis may aid in understanding the novel aspects of iNPH.
\end{abstract}

Correspondence to: Dr Yuki Nagata, Medical Genome Center, National Center for Geriatrics and Gerontology, 7-430 Morioka-cho, Obu, Aichi 474-8511, Japan

E-mail: nagata@ncgg.go.jp

Abbreviations: AD, Alzheimer's disease; CM, cerebral meninges; CSF, cerebrospinal fluid; DFNB84A, deafness autosomal recessive 84A; DiE, diencephalon; FNIII, fibronectin 3 domain; iNPH, idiopathic normal pressure hydrocephalus; PTP, protein tyrosine phosphatase; PTPRQ, protein tyrosine phosphatase receptor type Q; SNR, shunt non-responder; TM, transmembrane

Key words: idiopathic normal pressure hydrocephalus, shunt non-responder, diagnostic marker, cerebrospinal fluid, PTPRQ

\section{Introduction}

Idiopathic normal pressure hydrocephalus (iNPH) is caused by the accumulation of cerebrospinal fluid (CSF) and it leads to gait disturbance, urinary incontinence, and dementia (1). Bradley et al suggested that benign external hydrocephalus in infancy could exacerbate in late adulthood and might lead to iNPH $(1,2)$. Other groups have reported the existence of genetic predisposition to the disease $(3,4)$. However, the precise mechanism of onset of iNPH is still unknown. However, unlike other dementias, iNPH is treatable by shunt operation, which relieves the accumulated CSF (1).

In Japan, approximately $5 \%$ of dementia patients are diagnosed with iNPH. However, some symptoms of iNPH are similar to those of Alzheimer's disease (AD) or other dementias. Therefore, it is often difficult to distinguish iNPH from other dementias. Identifying biomarkers for iNPH is important to diagnose latent iNPH patients among dementia patients.

The objective of this study was to identify potential biomarkers for iNPH. To this purpose, we conducted a proteomic analysis of CSF of iNPH and AD patients using high-performance liquid chromatography (HPLC) coupled with electrospray ionization quadrupole time-of-flight mass spectrometry (LC-TOF-MS). We identified protein tyrosine phosphatase receptor type Q (PTPRQ) as a potential biomarker for iNPH.

$P T P R Q$ is known as a gene causing hearing loss, deafness autosomal recessive 84A (DFNB84A) (5,6). Goodyear et al reported that $P T P R Q$ null mice exhibit loss and fusion of cochlear stereocilia, resulting in hearing loss (7). Nayak et al indicated that the extracellular region of PTPRQ in the vestibular end organ is modified with dermatan sulfate. Depending on the location of the dermatan sulfate on PTPRQ, neighboring stereocilia adhere to or repel each other, thus maintaining a proper distance between the cilia (8).

In connection with these reports, patients with hydrocephalus-associated hearing loss are reported, and there are some reports on hearing loss related to shunt surgery (9-11). These reports suggest the relevance among iNPH, auditory capacity and PTPRQ. Therefore, in this study, we attempted to 
investigate the characteristics and concentration of PTPRQ in the CSF and the relation to auditory capacity.

\section{Materials and methods}

Subjects. Subjects with different diagnoses including healthy control, AD, iNPH and shunt non-responder (SNR) patients were examined. Subject characteristics are summarized in Table I. iNPH diagnosis was made according to the 'Guidelines for management of idiopathic normal pressure hydrocephalus: Second edition' (12). Shunt surgeries were done for probable iNPH patients and determined to be effective in case of 1 point or more improvement on the modified Rankin scale, or 2 points or more on the Japanese iNPH grading scale (13). $\mathrm{AD}$ diagnosis was made according to the published 'Research criteria for the diagnosis of Alzheimer's disease: Revising the NINCDS-ADRADA criteria' (14).

Bioresources were largely obtained from the BioBanks of the National Center for Geriatrics and Gerontology (NCGG, Aichi, Japan) and the National Center of Neurology and Psychiatry (NCNP, Tokyo, Japan) and partly donated by the Department of Biological Regulation, School of Medicine, Tottori University (Tottori, Japan). All samples were obtained with written informed consent from the patients before sampling between 2011 and 2015. This study was reviewed and approved by the Ethics Committees of the Institute and BioBanks.

Collection of CSF. CSF was collected by lumber puncture and centrifuged for $10 \mathrm{~min}$ at $800 \mathrm{xg}$ at $4^{\circ} \mathrm{C}$. The supernatant was aliquoted into low protein-binding tubes and immediately frozen in liquid nitrogen and stored at $-80^{\circ} \mathrm{C}$ until use.

Proteomic analysis by mass spectrometry. Four hundred microliters of CSF was concentrated by centrifugation using Ultra-4 Centrifugal Filters Ultracel-3K (Merck Millipore Japan, Tokyo, Japan) for $1 \mathrm{~h}$ at $4,000 \mathrm{xg}$ at $4^{\circ} \mathrm{C}$. Approximately $100 \mu \mathrm{l}$ of concentrated CSF was filtered through an Ultrafree-MC GV $0.22-\mu \mathrm{m}$ spin column (Merck Millipore Japan) for 2 min at $16,000 \mathrm{x} \mathrm{g}$ at $4^{\circ} \mathrm{C}$ to remove debris. CSF proteins were separated into high- and low-abundance proteins by HPLC using Human 14 Multiple Affinity Removal System Column (Agilent Technologies, Inc., Tokyo, Japan) according to the manufacturer's protocol. The low-abundance protein fraction was concentrated again and stored at $-80^{\circ} \mathrm{C}$ until analysis.

Proteomic analysis was conducted with LC-TOF-MS (QTOF Ultima; Nihon Waters K.K., Tokyo, Japan) as previously described (15-17). Briefly, samples were trypsin-digested and MS peaks were detected with LC-TOF-MS, followed by normalization and quantification with the 2DICAL software (Mitsui Knowledge Industry Co., Ltd., Tokyo, Japan) (18).

Enzyme-linked immunosorbent assay (ELISA). An ELISA kit for PTPRQ quantification was purchased from Cloud-Clone Corp. (Houston, TX, USA) and used according to the manufacturer's protocol. One hundred microliters of CSF was added per well. After quantification, the outliers more than median $\pm 3 \times$ SD were excluded from analysis.

Western blotting. Human total protein lysates of kidneys, brain, diencephalon (DiE), and cerebral meninges $(\mathrm{CM})$ were purchased from Cosmo Bio Co., Ltd. (Tokyo, Japan). Equal amounts of samples were mixed with Laemmli sample buffer (final volume containing 5\% $\beta$-mercaptoethanol), boiled for 3 min, and separated on a 7.5\% Mini-Protean TGX precast gel (Bio-Rad Laboratories, Tokyo, Japan). The separated proteins were transferred to a PVDF membrane using a Trans-Blot Turbo Transfer system (Bio-Rad Laboratories). The membrane was incubated with 5\% skim milk in Tris-buffered saline with Tween-20 (TBST) at room temperature for $1 \mathrm{~h}$ and then with rabbit polyclonal anti-PTPRQ antibody recognizing the extracellular domain (cat. no. PAD603Hu01, immunized with amino acids 36 to 294, 1:400 dilution; Cloud-Clone Corp.) or the intracellular domain (cat. no. sc-368569, immunized with amino acids 2208 to 2299, 1:1,000 dilution; Santa Cruz Biotechnology, Inc., Dallas, TX, USA) of PTPRQ in Can Get Signal 1 enhancer solution (Toyobo Co., Ltd., Osaka, Japan) at $4^{\circ} \mathrm{C}$, overnight. After washing thrice with TBST, the membrane was incubated with HRP-linked anti-rabbit IgG (cat. no. 7074; Cell Signaling Technology Japan, K. K., Tokyo, Japan) diluted to 1:2,000 in Can Get Signal 2 (Toyobo Co., Ltd.) and incubated at room temperature for $1 \mathrm{~h}$. After four washes with TBST, signal was detected with ImmunoStar LD (Wako Pure Chemical Industries, Ltd., Osaka, Japan) and an ImageQuant LAS-4000 imager (Fujitsu Ltd., Tokyo, Japan). The images were processed using MultiGauge software v3.2 (FujiFilm Corp., Tokyo, Japan). To improve visibility, we adjusted the exposure, contrast, and angle of the images.

Immunohistochemistry of the mouse brain tissue. The brain tissues were dissected from a male C57BL/6 mouse (8-week-old), fixed in $10 \%$ neutral formalin for $24 \mathrm{~h}$, embedded in paraffin, and cut into $2-\mu \mathrm{m}$ sections. Sections were dewaxed using xylene, rehydrated in a graded series of alcohol to water, and subjected to antigen retrieval using retrieval solution, pH 6.0 (Nichirei Biosciences, Inc., Tokyo, Japan) in a pressure cooker for $10 \mathrm{~min}$. Endogenous peroxidase was blocked by incubation of the brain sections with 3\% hydrogen peroxide for $10 \mathrm{~min}$. Sections were subsequently blocked with $3 \%$ bovine serum albumin (Wako Pure Chemical Industries, Ltd.) for $30 \mathrm{~min}$ to prevent non-specific antibody binding.

The sections were incubated with rabbit polyclonal $\operatorname{IgG}$ anti-PTPRQ (immunized with amino acids 2208 to 2299, 1:50 dilution; Santa Cruz Biotechnology, Inc.) for $60 \mathrm{~min}$, followed by incubation with the secondary anti-rabbit $\operatorname{IgG}$ antibody conjugated to horseradish peroxidase (HRP) (Nichirei Biosciences, Inc.) for $60 \mathrm{~min}$. Following each treatment, the slides were washed using phosphate-buffered saline (PBS; $3 \times 5$ min washes). Finally, they were stained using DAB. Sections were counterstained using Mayer's hematoxylin, dehydrated, cleared, and mounted.

Stained sections were analyzed under the microscope BX-53 (Olympus Corporation, Tokyo, Japan), and representative photographs were acquired using a digital camera DP21 (Olympus Corporation).

Hearing evaluation. The correlation between the amount of PTPRQ in the CSF and auditory capacity was investigated in 26 patients with iNPH who had undergone pure tone audiometry before the shunt operation. The patients included 8 males and 18 females (mean age, $79.9 \pm 3.6$; range, 72 to 88 ; median 
Table I. Subject characteristics.

\begin{tabular}{|c|c|c|c|c|c|c|}
\hline & \multicolumn{2}{|c|}{ LC-TOF-MS } & \multicolumn{4}{|c|}{ ELISA $^{a}$} \\
\hline & $\mathrm{AD}$ & $\mathrm{iNPH}^{\mathrm{b}}$ & Control & $\mathrm{AD}$ & $\mathrm{iNPH}^{\mathrm{c}}$ & SNR \\
\hline Subject no. & 7 & 8 & 12 & 48 & 36 & 8 \\
\hline Mean age (SD) & $71.3(7.7)$ & $75.3(4.5)$ & $71.2(9.6)$ & $75.5(8.0)$ & $79.6(5.1)$ & $78.9(3.0)$ \\
\hline Age range & $62-84$ & $70-84$ & $52-85$ & $54-87$ & $67-88$ & $76-84$ \\
\hline Male/female & $4 / 3$ & $5 / 3$ & $3 / 9$ & $20 / 28$ & $16 / 20$ & $3 / 5$ \\
\hline
\end{tabular}

LC-TOF-MS, liquid chromatography coupled with electrospray ionization quadrupole time-of-flight mass spectrometry; ELISA, enzyme-linked immunosorbent assay; AD, Alzheimer's disease; iNPH, idiopathic normal pressure hydrocephalus; SNR, shunt non-responder. ${ }^{\text {aSamples used }}$ in ELISA include all samples used in LC-TOF-MS. ${ }^{b}$ Four patients with iNPH (three males and one female), who did not undergo a shunt operation, are included in the iNPH category. 'Thirteen patients with iNPH (6 males and 7 females), who did not undergo a shunt operation, are included in the iNPH category.

PTPRQ concentration, $0.70 \pm 0.5$; range, 0.24 to $2.0 \mathrm{ng} / \mathrm{ml}$ ). The average threshold of right and left ear at frequencies of $125,500,1,000,2,000,4,000$, and $8,000 \mathrm{~Hz}$ was determined. Samples from all these patients were included in the ELISA.

Statistical analyses. For proteomic analysis and ELISA, data were analyzed using Welch's t-test in StatFlex version 6 (Artech Co., Ltd., Osaka, Japan). For hearing evaluation, the correlation between the amount of PTPRQ in the CSF and the pure tone audiometry was analyzed using a general linear model in the statistical analysis system (SAS) version 9.3 (SAS Institute Japan, Tokyo, Japan). A value of $\mathrm{P}<0.05$ was considered to indicate a statistically significant difference.

\section{Results}

The concentration of PTPRQ in CSF. We analyzed CSF samples from 7 AD and 8 iNPH patients (Table I) with LC-TOF-MS. We found 74 proteins showing a significantly different ion strength between AD and iNPH samples (7 were higher and 67 were lower in iNPH than in AD, data not shown). Among these, PTPRQ showed a P-value $<0.01$ (Fig. 1A).

To validate the concentration of PTPRQ, we conducted an ELISA using the same and additional samples of AD and iNPH patients. Additionally, we tested 12 healthy control and 8 SNR samples to evaluate the normal range of the PTPRQ concentration in the CSF and whether PTPRQ could distinguish SNRs among iNPH. The results confirmed that the PTPRQ level was significantly higher in iNPH than in healthy control and AD subjects (Fig. 1B). Furthermore, the median PTPRQ concentration in the CSF of SNRs was approximately 2 times lower than that in iNPH, albeit not significantly (Fig. $1 \mathrm{C}, \mathrm{P}=0.18$ ).

Qualitative analysis of PTPRQ by western blotting. In general, the ionic strength as determined by mass spectrometry is correlated with the relative abundance of the protein in the analyte. However, the abundances of PTPRQ estimated with mass spectrometry and ELISA were contradictory (Fig. 1A and B). In mass spectrometry, proteins are identified by quantifying the targets' molecular weights (19). Therefore, proteins having complex post-translational modifications occasionally fail to be detected. Thus, we presumed that PTPRQ in iNPH would have post-translational modifications different from that in AD. To verify this possibility, we performed western blotting with CSF samples from each of the diagnostic categories.

No obvious difference in PTPRQ band size was observed between iNPH patients and others (Fig. 2). This result indicated that PTPRQ in iNPH does not have a large difference in molecular weight as compared to the other diagnostic categories, or at least it was not detectable by western blotting. However, minor post-translational modifications could not be excluded.

Validation of PTPRQ expression in the brain tissues. Next, to confirm the expression of PTPRQ in the brain, we performed western blotting with total protein lysates from normal human tissues. Kidney lysate was used as a positive control (20). Further, we selected DiE and CM as well as whole-brain tissue lysates, which are all in contact with CSF, for analysis.

In kidney lysate, three main bands were observed (Fig. 3A and B), likely corresponding to the splicing variants depicted in Fig. 3C. Band $2(\sim 250 \mathrm{kDa})$ corresponds to the canonical isoform of PTPRQ (Uniprot ID Q9UMZ2), bands 1 $(>250 \mathrm{kDa})$ and $3(\sim 180 \mathrm{kDa})$ are likely isoforms with different numbers of the fibronectin 3 domain (FNIII) $(5,20)$. Band 4 ( $<150 \mathrm{kDa})$ was not observed in kidney lysate, but showed signal in whole-brain, DiE and CM. In these tissue lysates, all bands were detected at almost the same position by antibodies both detecting the extracellular (Fig. 3A) and the intracellular domain of PTPRQ (Fig. 3B). Band densities were stronger in $\mathrm{CM}$ than in brain and DiE samples, suggesting that PTPRQ is preferentially expressed in the CM. In CSF, the estimated molecular weight of the PTPRQ was approximately $170 \mathrm{kDa}$ when antibody for the extracellular domain of PTPRQ was used (Figs. 2 and 3A), whereas this band disappeared when antibody for the intracellular domain was used (Fig. 3B). These results suggested that PTPRQ in the CSF consists of part of the extracellular domain and is derived from the brain tissues.

Further, to examine the localization of PTPRQ in the brain, immunohistochemistry was performed using anti-PTPRQ antibody. The CM with arachnoid villi and DiE with third ventricle, which include choroid plexus, were chosen from the mouse. As a result, arachnoid villi and choroid plexus were found to be stained with anti-PTPRQ antibody (Fig. 4, arrows). 
A

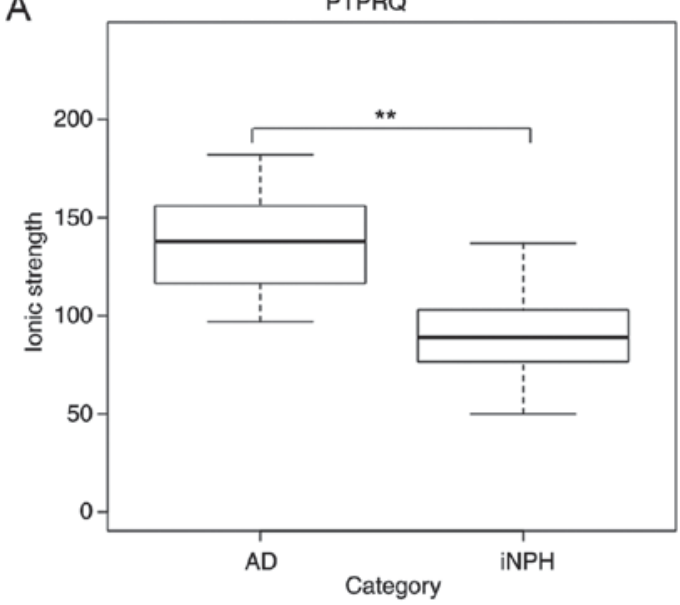

B

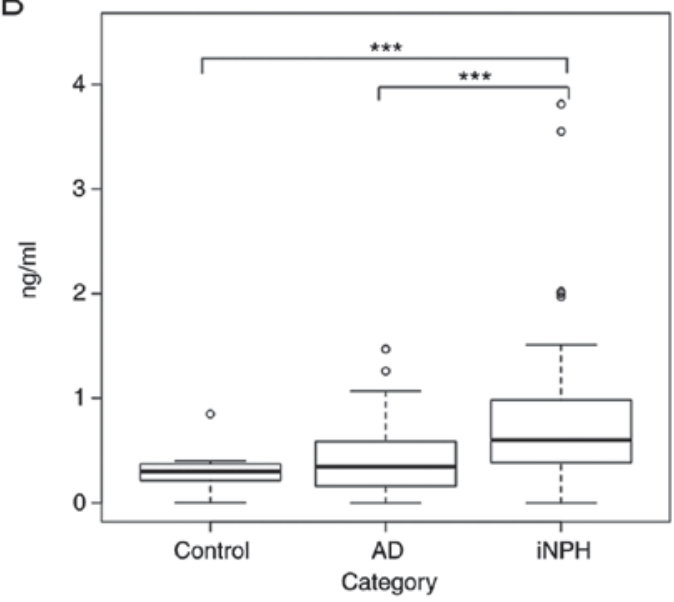

C

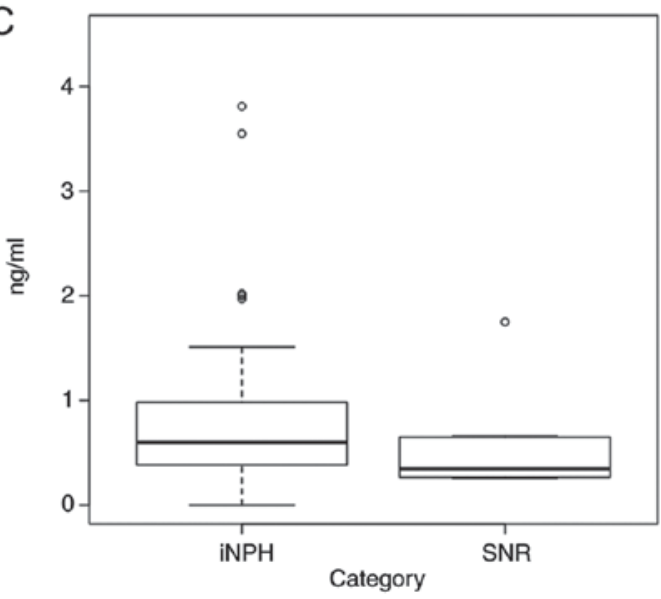

Figure 1. PTPRQ concentration in CSF. (A) The ionic strength of PTPRQ detected with LC-TOF-MS was higher in AD than in iNPH patients. $\mathrm{P}=0.007$ (Welch's t-test). (B) PTPRQ in CSF as tested by ELISA. Welch's t-test was used to compare groups. The P-value of control vs. iNPH was 0.0007 and that of AD vs. iNPH was 0.003 . Median values of control, AD, and iNPH were $0.30,0.35$, and $0.68 \mathrm{ng} / \mathrm{ml}$, respectively. (C) PTPRQ in CSF from iNPH and SNR are indicated. Welch's t-test was performed and the P-value was 0.18 . The middle line in the box represents the median, while the upper edge and lower edge represent the 1st quartile (1Q) and the 3rd quartile (3Q), respectively. Short whiskers indicate the minimum and maximum point of our data. The circles indicate outlier value. The potential outliers lie outside the range of $1 \mathrm{Q}-(\mathrm{IQRx} 1.5)$ to $3 \mathrm{Q}+(\mathrm{IQR} \times 1.5)$. IQR stands for interquartile range. ${ }^{* *} \mathrm{P}<0.01,{ }^{* * *} \mathrm{P}<0.005$. $\mathrm{PTPRQ}$, protein tyrosine phosphatase receptor type Q; AD, Alzheimer's disease; iNPH, idiopathic normal pressure hydrocephalus; SNR, shunt non-responder; CSF, cerebrospinal fluid; LC-TOF-MS, liquid chromatography coupled with electrospray ionization quadrupole time-of-flight mass spectrometry; ELISA, enzyme-linked immunosorbent assay.

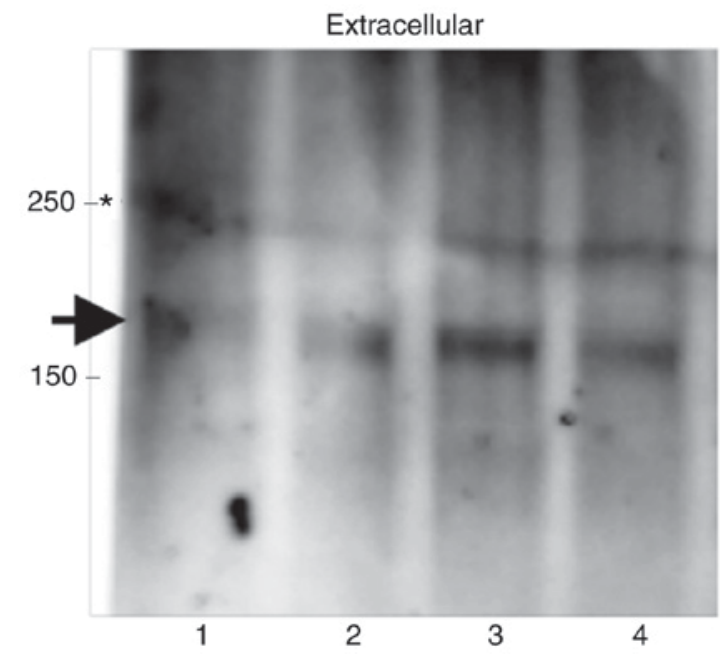

Figure 2. Estimation of the band size of PTPRQ in CSF. PTPRQ in CSF from healthy control (lane 1), AD patient (lane 2), iNPH patient (lane 3), and SNR (lane 4) were detected with the antibody for the extracellular domain. Same volumes of CSF were analyzed. The asterisk indicates a non-specific band near $250 \mathrm{kDa}$. PTPRQ, protein tyrosine phosphatase receptor type Q; CSF, cerebrospinal fluid; AD, Alzheimer's disease; iNPH, idiopathic normal pressure hydrocephalus; SNR, shunt non-responder.

Furthermore, the surface area of the third ventricle, where the ependymal cells along with cilia structure is observed and directly contacts the CSF, was immunostained with anti-PTPRQ antibody (Fig. 4B, asterisk). These results suggest that PTPRQ in the CSF comes in contact with the CSF from these structures.

Evaluation of hearing capability. Next, we investigated the potential correlation between the amount of PTPRQ in the CSF and auditory capacity by using a general linear model. We found no correlation between the amount of PTPRQ and the average hearing threshold at each frequency (Table II).

\section{Discussion}

Here, we report PTPRQ as a novel potential biomarker for iNPH. In the CSF of patients with iNPH, the PTPRQ concentration was significantly higher than in healthy controls and patients with AD. In addition, the median PTPRQ concentration in the CSF of SNRs was two times lower than that of patients with iNPH, although the difference was not significant.

As the abundances of PTPRQ estimated with mass spectrometry and ELISA were contradictory (Fig. 1A and B), we presumed PTPRQ in iNPH would have differential post-translational modifications compared to $\mathrm{AD}$ and performed western blotting. We found no obvious difference in PTPRQ size between iNPH patients and others (Fig. 2). However, there is a report indicating that transferrin in CSF from iNPH patients have a unique N-glycan modification (21), suggesting that PTPRQ in CSF from iNPH patients also possibly have unique modification. Unfortunately, PTPRQ in CSF is not abundant compared to transferrin, it makes difficult to analyze PTPRQ modifications at present. Further analyses with more accurate and high sensitive methods are needed.

Another western blotting indicated that the PTPRQ in the CSF consists of part of the extracellular domain of PTPRQ 
Table II. Pure tone audiometry results of patients.

\begin{tabular}{|c|c|c|c|c|c|c|c|}
\hline Frequency $(\mathrm{Hz})$ & 125 & 250 & 500 & 1,000 & 2,000 & 4,000 & 8,000 \\
\hline Mean $\pm \mathrm{SD}^{\mathrm{a}}$ & $36.9 \pm 14.5$ & $41.3 \pm 17.7$ & $41.9 \pm 16.0$ & $41.8 \pm 15.6$ & $59.0 \pm 16.6$ & $57.4 \pm 19.4$ & $71.0 \pm 19.8$ \\
\hline Correlation factor & -0.158 & -0.035 & 0.036 & 0.101 & -0.008 & -0.006 & -0.027 \\
\hline P-value & 0.441 & 0.865 & 0.863 & 0.623 & 0.969 & 0.975 & 0.897 \\
\hline
\end{tabular}

${ }^{a}$ Average threshold of right and left ear at each frequency.

A

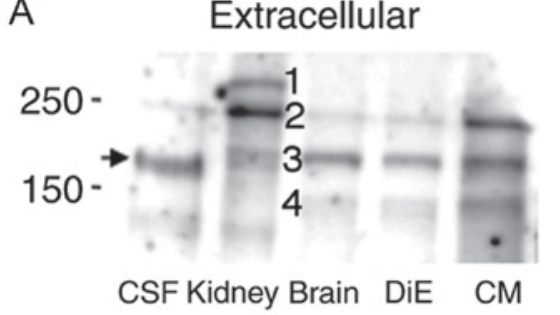

B

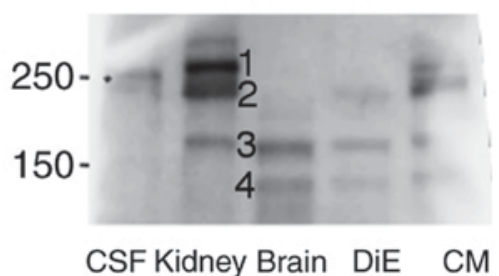

C

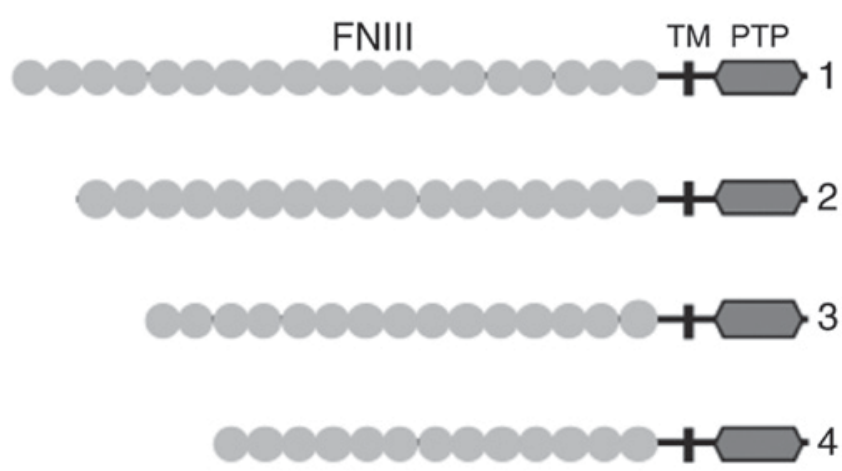

Figure 3. Western blotting of PTPRQ in the CSF and indicated tissue lysates. (A and B) Five microliters of CSF from iNPH patients and $25 \mu \mathrm{g}$ of total protein lysates from human kidney (positive control), brain, DiE, and CM tissues from healthy adults were analyzed. Western blots using antibodies recognizing (A) the extracellular and (B) the intracellular domain of PTPRQ are shown. (C) Schematic representations of the known $(1,2,3)$ and predicted $(4)$ isoforms of PTPRQ. Numbers correspond to the band numbers indicated on the western blot. PTPRQ, protein tyrosine phosphatase receptor type Q; CSF, cerebrospinal fluid; iNPH, idiopathic normal pressure hydrocephalus; DiE, diencephalon; CM, cerebral membrane; FNIII, fibronectin 3 domain; TM, transmembrane; PTP, protein tyrosine phosphatase. The asterisk indicates a non-specific band near $250 \mathrm{kDa}$.
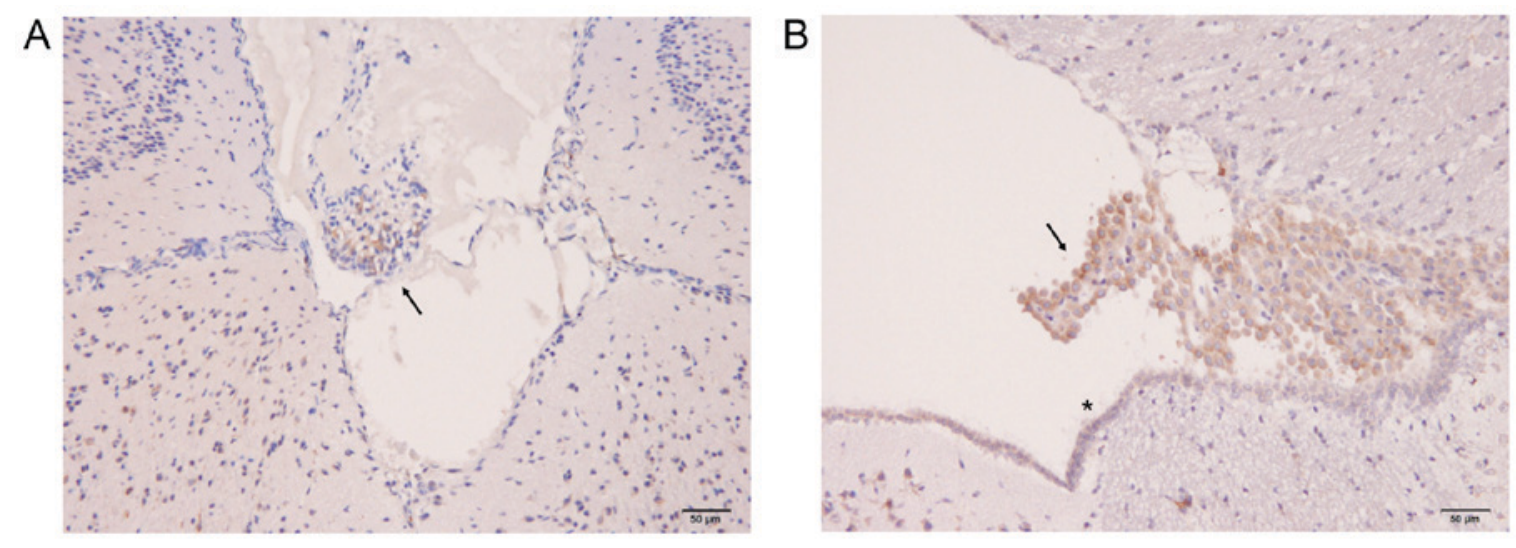

Figure 4. Immunohistochemistry of the mouse brain tissue using anti-PTPRQ antibodies. (A) Arachnoid villi in the mouse CM was immunostained with anti-PTPRQ antibody (arrow). (B) Choroid plexus (arrow) and the surface area of third ventricle (asterisk) were immunostained with anti-PTPRQ antibody. Scale bar, $50 \mu \mathrm{m}$. PTPRQ, protein tyrosine phosphatase receptor type Q; CM, cerebral meninges.

expressed in brain tissues. One intense band, which was detected only in the brain tissues (Fig. 3, band 4), did not correspond to any previously reported isoform of PTPRQ. However, PTPRQ isoforms have been studied only in kidney, 
retina, and testes (20). Band 4 may be an isoform unique to the brain tissue; we hypothesize it to be another FNIII-number variant. Additional immunohistochemistry of the mouse CM and DiE using anti-PTPRQ antibody revealed that PTPRQ is expressed at the site of CSF dynamics, arachnoid villi and choroid plexus, where the CSF is resorbed and produced, respectively (22). Furthermore, the surface area of the third ventricle, where the ependymal cells possessing the cilia structure, was immunostained with anti-PTPRQ antibody; these cilia structures are considered to have an important role in the CSF dynamics (23-25).

The physiological meaning of these findings remains unclear, though we suspect that PTPRQ might play a role in maintaining cilia structure in the brain tissues that are in contact with the CSF, the same as in the ear organ $(7,8)$. If so, the increase of PTPRQ fragments in the CSF might reflect a disruption of the ciliary structure in brain. Actually, some reports have indicated that genetic mutations in ciliary proteins produce congenital hydrocephalus (24,26-29). Thus, a disruption of the ciliary structure in brain possibly be one of the cause of iNPH. In the case of SNRs, the relatively low concentration of PTPRQ in the CSF might indicate progressed ciliary deficit, resulting in the exhaustion of PTPRQ protein. Alternatively, the disease in these patients might have another pathogenesis mechanism, without the increase of PTPRQ in the CSF. To evaluate these hypotheses, brain autopsies from iNPH patients are needed, though it is difficult to obtain at present.

We hypothesized PTPRQ in cochlear stereocillia would also be disrupted and cause hearing loss in iNPH patients. Thus, we expected a correlation between the auditory capacity and the amount of PTPRQ in the CSF of iNPH. However, no significant correlation was observed (Table II). It is speculated that PTPRQ disruption might be a locally restricted event in the brain of iNPH patients and hydrocephalus-associated hearing loss would be just a result of the pressure difference between CSF and inner ear fluids $(9,10,30)$. Although, our analysis was relatively small, further validation in lager sample size is required.

Usually, the diagnosis of iNPH is performed by CSF tap test, which removes several 10-ml aliquots of the CSF through a lumbar puncture. Therefore, the concentration of PTPRQ in the CSF of almost all the possible cases of iNPH could be readily assessed. We are expecting the accumulation of enough samples and the large-scale analysis would reveal the usefulness of PTPRQ as a marker for iNPH.

\section{Acknowledgements}

The present study was supported by the Program for Promotion of Fundamental Studies in Health Sciences of the National Institute of Biomedical Innovation of Japan (10-44) and The Research Funding for Longevity Sciences (26-20). We thank Dr Nobuyoshi Shimoda for helpful discussions. We wish to acknowledge Dr Takeshi Tomonaga for helpful advices on manuscript. We thank Editage (www.editage.jp) for English language editing.

\section{References}

1. Adams RD, Fisher CM, Hakim S, Ojemann RG and Sweet WH: Symptomatic occult hydrocephalus with normal cerebrospinal-fluid pressure. New Engl J Med 273: 117-126, 1965.
2. Bradley WG Jr, Bahl G and Alksne JF: Idiopathic normal pressure hydrocephalus may be a 'two hit' disease: Benign external hydrocephalus in infancy followed by deep white matter ischemia in late adulthood. J Magn Reson Imaging 24: 747-755, 2006.

3. Cusimano MD, Rewilak D, Stuss DT, Barrera-Martinez JC, Salehi F and Freedman M: Normal-pressure hydrocephalus: Is there a genetic predisposition? Can J Neurol Sci 38: 274-281, 2011.

4. Takahashi Y, Kawanami T, Nagasawa H, Iseki C, Hanyu H and Kato T: Familial normal pressure hydrocephalus (NPH) with an autosomal-dominant inheritance: A novel subgroup of NPH. J Neurol Sci 308: 149-151, 2011.

5. Schraders M, Oostrik J, Huygen PL, Strom TM, van Wijk E, Kunst HP, Hoefsloot LH, Cremers CW, Admiraal RJ and Kremer H: Mutations in PTPRQ are a cause of autosomal-recessive nonsyndromic hearing impairment DFNB84 and associated with vestibular dysfunction. Am J Hum Genet 86: 604-610, 2010.

6. Shahin H, Rahil M, Abu Rayan A, Avraham KB, King MC, Kanaan $\mathrm{M}$ and Walsh T: Nonsense mutation of the stereociliar membrane protein gene PTPRQ in human hearing loss DFNB84. J Med Genet 47: 643-645, 2010.

7. Goodyear RJ, Jones SM, Sharifi L, Forge A and Richardson GP: Hair-bundle defects and loss of function in the vestibular end organs of mice lacking the receptor-like inositol lipid phosphatase, PTPRQ. J Neurosci 32: 2762-2772, 2012.

8. Nayak G, Goodyear RJ, Legan PK, Noda M and Richardson GP: Evidence for multiple, developmentally regulated isoforms of Ptprq on hair cells of the inner ear. Dev Neurobiol 71: 129-141, 2011.

9. Dixon JF and Jones RO: Hydrocephalus-associated hearing loss and resolution after ventriculostomy. Otolaryngol Head Neck Surg 146: 1037-1039, 2012.

10. Sammons VJ, Jacobson E and Lawson J: Resolution of hydrocephalus-associated sensorineural hearing loss after insertion of ventriculoperitoneal shunt. J Neurosurg Pediatr 4: 394-396, 2009.

11. van Veelen-Vincent ML, Delwel EJ, Teeuw R, Kurt E, de Jong DA, Brocaar MP, Pauw BK, Avezaat CJ and van Zanten BG: Analysis of hearing loss after shunt placement in patients with normal-pressure hydrocephalus. J Neurosurg 95: 432-434, 2001.

12. Mori E, Ishikawa M, Kato T, Kazui H, Miyake H, Miyajima M, Nakajima M, Hashimoto M, Kuriyama N, Tokuda T, et al: Guidelines for management of idiopathic normal pressure hydrocephalus: Second edition. Neurol Med Chir (Tokyo) 52: 775-809, 2012.

13. Kubo Y, Kazui H, Yoshida T, Kito Y, Kimura N, Tokunaga H, Ogino A, Miyake H, Ishikawa M and Takeda M: Validation of grading scale for evaluating symptoms of idiopathic normal-pressure hydrocephalus. Dement Geriatr Cogn Disord 25: 37-45, 2008.

14. Dubois B, Feldman HH, Jacova C, Dekosky ST, Barberger-Gateau P, Cummings J, Delacourte A, Galasko D, Gauthier S, Jicha G, et al: Research criteria for the diagnosis of Alzheimer's disease: Revising the NINCDS-ADRDA criteria. Lancet Neurol 6: 734-746, 2007.

15. Matsubara J, Ono M, Negishi A, Ueno H, Okusaka T, Furuse J, Furuta K, Sugiyama E, Saito Y, Kaniwa N, et al: Identification of a predictive biomarker for hematologic toxicities of gemcitabine. $\mathrm{J}$ Clin Oncol 27: 2261-2268, 2009.

16. Ono M, Matsubara J, Honda K, Sakuma T, Hashiguchi T, Nose H, Nakamori S, Okusaka T, Kosuge T, Sata N, et al: Prolyl 4-hydroxylation of alpha-fibrinogen: A novel protein modification revealed by plasma proteomics. J Biol Chem 284: 29041-29049, 2009.

17. Takakura M, Yokomizo A, Tanaka Y, Kobayashi M, Jung G, Banno M, Sakuma T, Imada K, Oda Y, Kamita M, et al: Carbonic anhydrase I as a new plasma biomarker for prostate cancer. ISRN Oncol 2012: 768190, 2012.

18. Ono M, Shitashige M, Honda K, Isobe T, Kuwabara H, Matsuzuki H, Hirohashi S and Yamada T: Label-free quantitative proteomics using large peptide data sets generated by nanoflow liquid chromatography and mass spectrometry. Mol Cell Proteomics 5: 1338-1347, 2006.

19. Mann M, Højrup P and Roepstorff P: Use of mass spectrometric molecular weight information to identify proteins in sequence databases. Biol Mass Spectrom 22: 338-345, 1993.

20. Seifert RA, Coats SA, Oganesian A, Wright MB, Dishmon M, Booth CJ, Johnson RJ, Alpers CE and Bowen-Pope DF: PTPRQ is a novel phosphatidylinositol phosphatase that can be expressed as a cytoplasmic protein or as a subcellularly localized receptor-like protein. Exp Cell Res 287: 374-386, 2003. 
21. Futakawa S, Nara K, Miyajima M, Kuno A, Ito H, Kaji H, Shirotani K, Honda T, Tohyama Y, Hoshi K, et al: A unique $\mathrm{N}$-glycan on human transferrin in CSF: A possible biomarker for iNPH. Neurobiol Aging 33: 1807-1815, 2012.

22. McComb JG: Recent research into the nature of cerebrospinal fluid formation and absorption. J Neurosurg 59: 369-383, 1983.

23. Narita K and Takeda S: Cilia in the choroid plexus: Their roles in hydrocephalus and beyond. Front Cell Neurosci 9: 39, 2015

24. Banizs B, Pike MM, Millican CL, Ferguson WB, Komlosi P, Sheetz J, Bell PD, Schwiebert EM and Yoder BK: Dysfunctional cilia lead to altered ependyma and choroid plexus function, and result in the formation of hydrocephalus. Development 132: 5329-5339, 2005.

25. Siyahhan B, Knobloch V, de Zélicourt D, Asgari M, Schmid Daners M, Poulikakos D and Kurtcuoglu V: Flow induced by ependymal cilia dominates near-wall cerebrospinal fluid dynamics in the lateral ventricles. J R Soc Interface 11: 20131189, 2014.
26. Ibañez-Tallon I, Pagenstecher A, Fliegauf M, Olbrich H, Kispert A, Ketelsen UP, North A, Heintz N and Omran H: Dysfunction of axonemal dynein heavy chain Mdnah5 inhibits ependymal flow and reveals a novel mechanism for hydrocephalus formation. Hum Mol Genet 13: 2133-2141, 2004.

27. Lechtreck KF, Delmotte P, Robinson ML, Sanderson MJ and Witman GB: Mutations in Hydin impair ciliary motility in mice. J Cell Biol 180: 633-643, 2008

28. Lee L: Riding the wave of ependymal cilia: Genetic susceptibility to hydrocephalus in primary ciliary dyskinesia. J Neurosci Res 91: 1117-1132, 2013.

29. Tissir F, Qu Y, Montcouquiol M, Zhou L, Komatsu K, Shi D, Fujimori T, Labeau J, Tyteca D, Courtoy P, et al: Lack of cadherins Celsr2 and Celsr3 impairs ependymal ciliogenesis, leading to fatal hydrocephalus. Nat Neurosci 13: 700-707, 2010.

30. Gordon AG: Endolymphatic hydrops and CSF pressure. J Neurosurg 60: 1332-1334, 1984. 